\title{
Enfermedad de Lyme: a propósito de dos casos
}

\author{
A. Pérez Guirado ${ }^{a}$, R. I. Fernández Fernández ${ }^{a}$, E. Arbesu Fernández ${ }^{b}$, P. M. Santos Rodríguez ${ }^{a}$
}

aPediatra. CS de Cangas del Narcea. Cangas del Narcea, Asturias. España • b Técnico de Salud. Hospital Carmen y Severo Ochoa. Cangas del Narcea, Asturias. España.

Publicado en Internet:

13-septiembre-2013

Alejandro Pérez Guirado: rofesa2006@hotmail.com

Palabras clave:
- Enfermedad de Lyme

- Eritema crónico migrans

- Tratamiento

- Parálisis facial

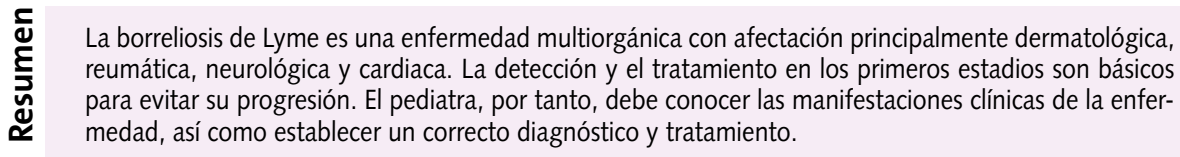
Se exponen dos casos que incluyen las formas más frecuentes de presentación.

\section{Lyme disease: report of two cases}

\footnotetext{
Key words:

- Lyme disease

- Erythema chronicum migrans

- Therapeutics

- Facial paralysis

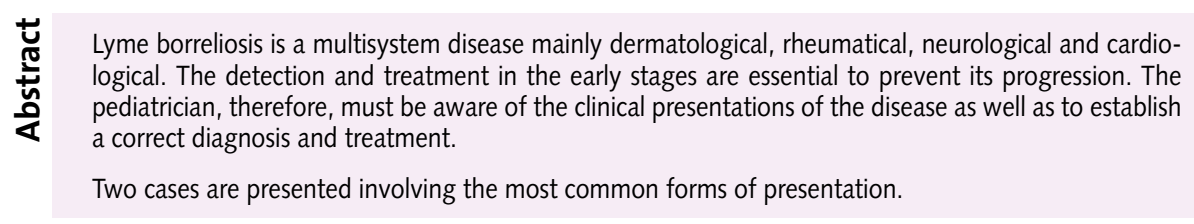

\section{INTRODUCCIÓN}

La borreliosis (o enfermedad) de Lyme es una enfermedad compleja con manifestaciones cutáneas, articulares, neurológicas, cardiacas y otras manifestaciones sistémicas, cuya lesión más específica desde el punto de vista diagnóstico es el eritema crónico migratorio $(E M)^{1}$ que resulta de la infección por espiroquetas del complejo Borrelia burgdorferi sensu lato ${ }^{2}$. En Europa, este complejo incluye tres genoespecies patógenas: Borrelia burgdorferi sensu stricto, Borrelia afzelii y Borrelia garinii ${ }^{1,2}$.

Su vector principal, la garrapata Ixodes ricinus, se encuentra ampliamente distribuido en la Península ibérica ${ }^{1}$. Se trata de la infección transmitida por un vector más frecuente en Norteamérica y Europa ${ }^{2}$.

La distribución de esta garrapata a nivel mundial hace de la borreliosis de Lyme una enfermedad asociada a áreas forestales de hoja caduca del he-

Cómo citar este artículo: Pérez Guirado A, Fernández Fernández RI, Arbesu Fernández E, Santos Rodríguez PM. Enfermedad de Lyme: a propósito de dos casos. Rev Pediatr Aten Primaria. 2013;15:249.e105-e109. 
misferio norte ${ }^{3}$. La cornisa cantábrica y el nordeste de España están ampliamente pobladas por este artrópodo. Se han encontrado Ixodes en Galicia, Asturias, Cantabria, País Vasco y Castilla y León, en la zona este (Cataluña), en la zona centro (Madrid) y en el sur (Andalucía). Zonas del norte de España como La Rioja, Navarra o la sierra del Sueve en Asturias son zonas endémicas de borreliosis de Lyme ${ }^{1}$. Debido al ciclo vital de la garrapata, la enfermedad suele iniciarse durante los meses de junio, julio y agosto. Las ninfas pueden estar activas durante todo el año en zonas húmedas y templadas como la cornisa cantábrica ${ }^{1}$. La transmisión suele producirse tras 48-72 horas del anclaje de la garrapata ${ }^{2}$. Las manifestaciones clínicas dependen del estadio de la enfermedad ${ }^{3}$ :

- Enfermedad temprana localizada: eritema migrans o migratorio (EM).

- Enfermedad temprana diseminada: EM múltiple, parálisis de nervios craneales (especialmente del nervio facial), meningitis, carditis.

- Enfermedad tardía: artritis, encefalopatía o polineuropatía subaguda.

Los primeros signos de infección se hacen evidentes semanas o pocos meses después de la picadura, mientras que los tardíos lo hacen muchos meses, e incluso años, después. Las manifestaciones tempranas suelen ser autolimitadas, mientras que las tardías se pueden hacer crónicas e incluso generar daño orgánico irreversible 3 .

Durante las primeras fases de la enfermedad temprana localizada (primeras cuatro semanas), los test serológicos no tienen suficiente sensibilidad para ayudar en el diagnóstico, por lo que el conocimiento de la clínica es fundamental ${ }^{4-6}$. En caso de solicitar estudios complementarios, lo más adecuado es el llamado enfoque de dos pasos mediante una cuantificación de anticuerpos mediante enzimoinmunoanálisis (EIA) en primer lugar y, en caso de positividad o resultado indeterminado, realizar un estudio, más sensible y específico, por inmunotransferencia de Western ${ }^{4-7}$. Cuando se investiga la enfermedad temprana diseminada, deben efectuarse EIA para IgG e IgM; cuando se sospecha una enfermedad tardía, solo para $\lg ^{5-7}$. No se recomienda la realización de serologías ante síntomas inespecíficos, dada la posibilidad de falsos positivos ${ }^{4-7}$.

En la enfermedad temprana localizada, la doxiciclina es el tratamiento de elección en mayores de ocho años de edad (Tabla 1). Los niños más pequeños se tratan con amoxicilina oral. La cefuroxima es un fármaco alternativo. La eritromicina y la azitromicina son menos eficaces ${ }^{6,8}$.

Por último, son útiles medidas preventivas como llevar pantalones largos o la aplicación de repelentes. El examen cutáneo en busca de garrapatas es recomendable aunque de efectividad dudosa, dada facilidad de que pasen desapercibidas por su pequeño tamaño. Una vez localizada, la garrapata debe ser extraída mediante una pequeña pinza, lo más cerca posible de su cabeza y evitando la compresión de su cuerpo, la rotación del mismo o la aplicación de agentes químicos tópicos ${ }^{4}$. No está indicada la profilaxis antibiótica tras la picadura, aun en zonas endémicas ${ }^{4,6}$.

\section{CASO 1}

Paciente varón de 13 años que acude a la consulta por cervicalgia de 20 días de evolución, no irradiada y sin alteraciones sensoriales o motoras en los miembros superiores, de predominio nocturno y con mala respuesta al tratamiento con ibuprofeno y diazepam. Diplopia intermitente, fiebre de dos días de duración al inicio del proceso, sin síntomas generales acompañantes. A los diez días del inicio de la clínica se realizan hemograma, velocidad de sedimentación globular y radiografía cervical, que son normales.

En la exploración física destaca una desviación de la comisura bucal con la sonrisa; el resto de la exploración neurológica es normal. No presenta exantemas, aunque la madre refiere, al ser preguntada, una picadura en un hombro dos meses antes, con eritema circular progresivo de unas dos semanas de duración.

Ante la sospecha de enfermedad de Lyme, se rea- 


\begin{tabular}{|c|c|c|}
\hline & Categoría de la enfermedad & Fármaco y dosis \\
\hline \multirow[t]{3}{*}{$\begin{array}{l}\text { Enfermedad temprana } \\
\text { localizada }\end{array}$} & Ocho años de edad o mayores & $\begin{array}{l}\text { Doxiciclina: } 100 \mathrm{mg} \text {, vía oral, cada } 12 \text { horas, } \\
\text { durante 14-21 días }\end{array}$ \\
\hline & Menores de ocho años & $\begin{array}{l}\text { Amoxicilina: } 50 \mathrm{mg} / \mathrm{kg} / \mathrm{día} \text {, vía oral, en tres dosis, } \\
\text { durante } 14-21 \text { días }\end{array}$ \\
\hline & Pacientes que no toleren amoxicilina & $\begin{array}{l}\text { Cefuroxima: } 30 \mathrm{mg} / \mathrm{kg} / \text { día, vía oral, en dos dosis, } \\
\text { durante } 14-21 \text { días }\end{array}$ \\
\hline \multirow[t]{5}{*}{$\begin{array}{l}\text { Enfermedad temprana } \\
\text { diseminada y tardía }\end{array}$} & Parálisis facial aislada & $\begin{array}{l}\text { Mismo régimen que en la enfermedad temprana } \\
\text { localizada, pero durante } 21-28 \text { días }\end{array}$ \\
\hline & Artritis & $\begin{array}{l}\text { Mismo régimen que en la enfermedad temprana } \\
\text { localizada, pero durante } 28 \text { días }\end{array}$ \\
\hline & Artritis persistente o recurrente & $\begin{array}{l}\text { Una de las siguientes opciones: } \\
\text { - Ceftriaxona: } 75-100 \mathrm{mg} / \mathrm{kg} \text {, IV/IM, cada } 24 \text { horas, } \\
\text { durante } 14-28 \text { días } \\
\text { - Penicilina G: } 300000 \mathrm{U} / \mathrm{kg} / \text { día, IV, en dosis } \\
\text { divididas cada cuatro horas, durante } 14-28 \text { días } \\
\text { - Mismo régimen oral que en la enfermedad } \\
\text { temprana }\end{array}$ \\
\hline & Carditis & $\begin{array}{l}\text { Ceftriaxona o penicilina como en la artritis } \\
\text { persistente o recurrente }\end{array}$ \\
\hline & Meningitis o encefalitis & $\begin{array}{l}\text { Ceftriaxona o penicilina como en la artritis } \\
\text { persistente o recurrente, pero durante } 14-28 \text { días } \\
\text { (la ceftriaxona debe administrarse IV) }\end{array}$ \\
\hline
\end{tabular}

IM: vía intramuscular; IV: vía intravenosa.

Fuente: Red Book, $2009^{6}$.

lizan estudios serológicos con EIA (IgG, IgM) e inmunotransferencia de Western (IgG, IgM), ambos positivos, tras lo cual se inicia tratamiento con doxiciclina oral durante 21 días. Visto en consulta un mes tras el tratamiento, el paciente se encuentra asintomático.

\section{CASO 2}

Paciente varón de siete años de edad con exantema axilar no pruriginoso de 15 días de duración y crecimiento progresivo, sin otros síntomas asociados. Refiere picaduras de garrapata a nivel axilar y en una pierna un mes antes de la consulta.

La exploración muestra una lesión anular eritematosa de bordes irregulares, sin descamación, de unos $15 \mathrm{~cm}$ de diámetro (Fig. 1). Ante la sospecha de EM se inicia tratamiento con amoxicilina oral (21 días) y se realizan estudios serológicos con EIA (IgG, IgM) e inmunotransferencia de Western (IgG, IgM), que resultaron positivos. A los cinco días de iniciado el tratamiento desaparece el eritema.

\section{DISCUSIÓN}

El diagnóstico de la enfermedad es fácil cuando el médico conoce la entidad, se acompaña del típico EM y se refieren antecedentes de picadura de garrapata.

La característica más precoz de la enfermedad de Lyme, y la más frecuente en la infancia, es una erupción cutánea única denominada $E M$, que se presenta hasta en un $90 \%$ de los niños afectados ${ }^{2,6,9}$. Aparece en el lugar de la inoculación aproximadamente a los 7-14 días de la picadura (rango 3-30 días), aunque la lesión puede aparecer en un lugar diferente al sitio recordado, dada la posibilidad de picaduras no reconocidas ${ }^{3}$. Una historia de picadura de garrapata en un pasado más lejano debería considerarse una evidencia de riesgo potencial de exposición, mientras que no recordar una picadura no debe descartar el diagnóstico ${ }^{3}$. La manifestación inicial suele ser una mácula o pápula roja en el lugar de la picadura que después se ensancha lentamente ad- 


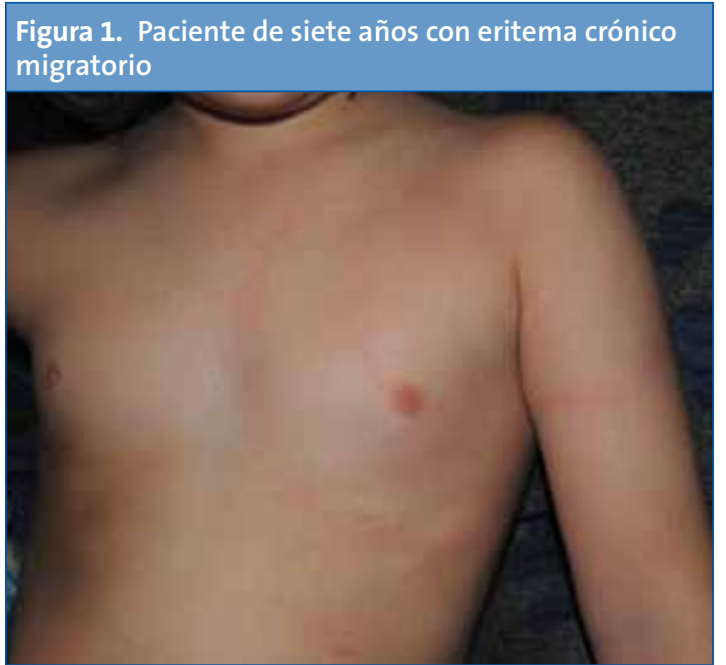

quiriendo forma de anillo irregular de borde rojo no sobreelevado ${ }^{2,3}$. Otras veces se presenta de forma más atípica como una placa edematosa, roja y caliente, incluso podrían aparecer vesículas o áreas de necrosis en la zona central de la lesión en relación con la intensa respuesta inflamatoria que se produce ${ }^{2,6}$. Suele ser indolora y durar de días a semanas adquiriendo un tamaño considerable $^{3}$.

La lesión es más frecuente en la cabeza y el cuello en niños pequeños, y en las extremidades en niños mayores ${ }^{3}$. A menudo asocia síntomas como cefalea, fatiga, mialgias, artralgias, dolor cervical, rigidez nucal, fiebre, náuseas, vómitos, dolor de garganta o linfadenopatía 2,3,6,9.

El diagnóstico diferencial incluye entidades como hipersensibilidad a la picadura de insectos, infección cutánea, urticaria, eritema multiforme, eritema anular centrífugo, granuloma anular o tinea corporis, basándose en la morfología, el tamaño, el prurito, la localización, el dolor o los cambios secundarios, como por ejemplo descamación ${ }^{3,4}$.

Si la enfermedad de Lyme no es reconocida y tratada en esta fase inicial, las espiroquetas penetran en el torrente sanguíneo y se diseminan por diferentes órganos y tejidos produciendo las siguientes etapas de la enfermedad, lo cual hace tan importante su sospecha ante la aparición de un exantema compatible ${ }^{3,6}$.
La manifestación más frecuente de la neuroborreliosis de Lyme en Europa es la parálisis del nervio facial, ante la cual se debe pensar siempre en un Lyme, sobre todo si es bilateral ${ }^{5,9,10}$. Usualmente acontece 1-12 (principalmente 4-6) semanas tras la picadura ${ }^{5}$. Hasta en un $80 \%$ de los casos asocia manifestaciones sistémicas como náuseas, dolor abdominal, artralgia y cefalea ${ }^{10}$. Son predictores independientes de enfermedad de Lyme como causa el inicio durante la estación epidémica, la ausencia de lesión herpética previa, la presencia de fiebre y la historia de cefalea. Los pacientes con estos tres predictores tienen un 50\% de riesgo de presentar enfermedad de Lyme y sería aconsejable la realización de estudios serológicos y considerar el inicio del tratamiento ante la presencia de más de uno de ellos 5,10

La mayoría de los pacientes con una parálisis facial aislada pueden ser tratados exitosamen-

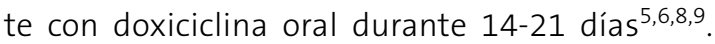
No hay evidencia de que los niños con cualquier manifestación de la enfermedad se beneficien de tratamientos antibióticos prolongados, por lo que la duración máxima de los mismos no debe sobrepasar las cuatro semanas ${ }^{6}$. En este caso, el tratamiento no busca la resolución de la parálisis facial, cuyo pronóstico es excelente, sino prevenir la enfermedad tardía, y no deben administrarse corticosteroides ${ }^{8}$.

La realización de una punción lumbar es controvertida, aunque podría ser innecesaria cuando no existe clínica de afectación del sistema nervioso central, como rigidez nucal, edema de papila o cefalea intensa o prolongada, ya que el significado clínico de las alteraciones en el líquido cefalorraquídeo es incierta y la evolución con el tratamiento oral excelente ${ }^{3,6}$. La mayoría de los pacientes son seropositivos en este estadio ${ }^{7}$. Se debe ser precavido al interpretar el resultado de los test diagnósticos. En ambos casos clínicos la sospecha diagnóstica inicial es fundamental, teniendo en cuenta que un EIA y una inmunotransferencia de Western positivos no indican necesariamente enfermedad de Lyme, al igual que un resultado negativo no la descarta por completo ${ }^{5}$. En áreas endémicas, ante 
una clínica inespecífica, el valor predictivo positivo del test es pobre y, por otro lado, en pacientes con enfermedad previa puede persistir seropositividad incluso tras un tratamiento antibiótico adecuado y una recuperación completa ${ }^{4,5}$.

Por último, llama la atención que, frente a una incidencia en España estimada de 0,25 casos por 100000 habitantes $^{11}$, desde el Servicio de Microbiología del Hospital Carmen y Severo Ochoa, hospital de referencia de nuestra área sanitaria en el suroccidente asturiano, se refiere una incidencia de 130 casos por 100000 habitantes, pudiendo considerarse pues endémica en el área. Por otro

\section{BIBLIOGRAFÍA}

1. Guerrero A. Borreliosis de Lyme en España. Enferm Infecc Microbiol Clin. 2001;19:244-6.

2. Bhate Ch, Schwartz R. Lyme disease advances and perspectives. J Am Acad Dermatol. 2011;64:619-36.

3. Shapiro ED. Lyme disease:clinical manifestations in children. UpToDate [en línea] [consultado en abril de 2013]. Disponible en www.uptodate.com

4. Bhate Ch, Schwartz R. Lyme disease management and prevention. J Am Acad Dermatol. 2011;64:63953.

5. Mygland A, Ljøstad U, Fingerle V. EFNS guidelines on the diagnosis and management of European Lyme neuroborreliosis. Eur J Neurol. 2010;17:8-16.

6. American Academy of Pediatrics. Lyme, enfermedad de. En: Pickering LK, Baker CJ, Long SS, McMillan JA lado, solo se han comunicado los dos casos pediátricos señalados en este artículo, por lo que es probable que esté infradiagnosticada en estas edades, bien porque no se consulta o bien por desconocimiento clínico.

\section{CONFLICTO DE INTERESES}

Los autores declaran no presentar conflictos de intereses en relación con la preparación y publicación de este artículo.

\section{ABREVIATURAS}

EIA: enzimoinmunoanálisis • EM: eritema migratorio. tría, 28. e ed. Madrid: Editorial Médica Panamericana; 2009. p. 474-9.

7. Linden H. Diagnosis of Lyme disease. UpToDate [en línea] [consultado en abril de 2013]. Disponible en www.uptodate.com

8. Linden $\mathrm{H}$. Treatment of Lyme disease. UpToDate [en línea] [consultado en abril de 2013]. Disponible en www.uptodate.com

9. O'Connell S. Lyme borreliosis: current issues in diagnosis and management. Curr Opin Infect Dis. 2010;23:231-5.

10. Lorch M, Teach JS. Facial nerve palsy etiology and approach to diagnosis and treatment. Pediatr Emer Care. 2010;26:763-72.

11. Escudero-Nieto R, Guerrero-Espejo A. Enfermedades producidas por Borrelia. Enferm Infecc Microbiol Clin. $2005 ; 23: 232-40$ 